\title{
In Silico Investigation of the Functional Impact of SCN10A Mutations in Human Atrial Cells
}

\author{
Inas Ali AL Nemi ${ }^{1}$, Haibo $\mathrm{Ni}^{1,2}$, Henggui Zhang ${ }^{1}$ \\ ${ }^{1}$ The University of Manchester, Manchester, UK \\ ${ }^{2}$ University of California Davis, Davis, CA, USA
}

\begin{abstract}
In normal conditions, action potential (AP) in cardiac myocytes is initiated by peak sodium current $I_{N a P}$, which become inactivated during depolarization, leaving a less pronounce current known as late sodium current $\left(I_{N a L}\right)$, which has a relatively smaller amplitude ( 0.5\%) compared to the $I_{N a P}$. A recent study has identified three gain-of-function mutations in the SCN10A channel (the gene encoding the voltage-gated sodium channel $\mathrm{Na}_{v} 1.8$, $I_{N a L}$ ) that have a correlation with the subsequent development of atrial fibrillation. However, the underlying mechanism responsible for the pro-arrhythmic effect of the gene mutations is unclear. This study aims to investigate the effect of the three gene mutations on atrial electrical action potentials. Hodgkin-Huxley (HH) formulation of late sodium channel current in human atrial cells was developed and validated against experimental data obtained by voltage clamp techniques. The formulation was then implemented in Colman et al. human atrial model to simulate atrial APs for wild type (WT) and mutation conditions. It was shown that A1073 and P1092 mutations prolong the action potential duration (APD) and elevate the plateau (phase 2) potential compared to WT. Such altered AP profiles may alter tissue's spatial heterogeneity, favoring the initiation and maintenance of atrial fibrillation, which warrants future study.
\end{abstract}

\section{Introduction}

At the beginning of depolarization of cell membrane to generate an action potential (AP), sodium channels activate producing the peak current $\left(I_{N a P}\right)$ that is responsible for generating the rapid upstroke (Phase 0 ) of the AP. During a short time window (in the range of several milliseconds), some of the $\mathrm{Na}^{+}$channels are inactivated and the sodium current starts to decay, leaving a small $I_{N a}$ during the rest time course of AP. However, there is a residual sodium current, $I_{N a L}$, which contributes to Phase 2 of AP along with the L-type calcium current $\left(I_{C a L}\right)$ [1].

Although $I_{N a L}$ is less pronounced in modulating the AP of cardiac myocytes, it could be enhanced under different pathological conditions such as $\mathrm{AF}$, heart failure and ischemia [1-3]. Moreover, a considerable increase in $I_{N a L}$ may result in an abnormality in the inactivation gate, which has distinctive mechanisms and causes. Therefore, $I_{N a L}$ is attracting more attention as a desirable element in pathology and pharmacology for treating arrhythmias [5].

The link between atrial fibrillation (AF) (one of the most common type of cardiac arrhythmia) and mutation in different ion channels have been investigated in some previous studies [6-9]. Genome-wide association studies (GWAS) have identified the influence on the PR-interval duration related to genetic variants in $S C N 10 A$. Ritchie et al. have reported that $\mathrm{SCN} 10 \mathrm{~A}$ variants have a direct correlation with the subsequent development of $\mathrm{AF}$ [10].

In a recent study [11], three gain-of-function mutations (R1588Q, A1073, and P1092) in the SCN10A gene have been identified in patients with familial AF. However, the causative link between the identified mutations and proarrhythmogenesis in human atria has not been established yet. This study aimed to investigate the functional impact of the identified genetic variations related SCN10A channel on the electrical APs of human atrial cells.

\section{Methods}

Hodgkin-Huxley formulations of the $I_{N a L}$ developed by Grandi et al. [12] for human atrial cell were adopted and then incorporated into the modified version of Colman et al. model for the electrical APs of human atrial cells [13]. In simulation, the Nelder-Mead simplex algorithm was used to obtain the optimal set of parameters of the model that reproduce relatively the same kinetics of the $I_{N a L}$ as obtained from the whole-cell patch clamp study of SCN10A channel for the wild type (WT), R1588Q, A1073, and P1092 mutations [11]. To validate the $I_{N a L}$ model equation, simulated current-voltage relationship (I-V curve) were compared to experimental data as shown in Figure 1. The conductance of the WT $I_{N a L}$ was chosen to be $0.5 \%$ of that of $I_{N a P}$ illustrated in literature $[14,15]$. Specifically, the model of the $I_{N a L}$ was given by the following equation: 


$$
I_{N a L}=\mathrm{G}_{\mathrm{NaL}} \times \mathrm{m}_{\mathrm{L}}^{3}\left(\mathrm{~A}_{\mathrm{f}} \times \mathrm{h}_{\mathrm{L}}+\mathrm{A}_{\mathrm{s}} \times \mathrm{j}_{\mathrm{L}}\right)\left(\mathrm{V}_{\mathrm{m}}-\mathrm{E}_{\mathrm{Na}}\right)
$$

where $\mathrm{G}_{\mathrm{NaL}}$ is the maximum conductance of the channel, $m_{L}$ is the activation gate, $h_{L}$ and $j_{L}$ are the fast and slow inactivation gates respectively, $A_{f}$ and $A_{s}$ are the fractions of fast and slow inactivation gates respectively, $V_{m}$ is the membrane potential, and $\mathrm{E}_{\mathrm{Na}}$ is the reversal potential of the channel.

The $I_{N a L}$ was then included to the total ionic current $I_{\text {ion }}$ that describe the change in the membrane potential:

$$
\frac{d V_{m}}{d t}=-\frac{I_{\text {ion }}}{C_{m}}
$$

where $V_{m}$ and $C_{m}$ are the membrane potential and capacitance respectively. This equation can be integrated to obtain the AP.

To generate the electrical APs, the atrial single cell model was paced at the basic cycle length (BCL) of 1000 $\mathrm{ms}$ for $30 \mathrm{~s}$ to ensure the steady-state was reached before AP recorded for analysis. The effects of the SCN10A channel mutations on the AP morphology and duration were investigated. In addition, the impact of the genetic variation on the APD and plateau potential were quantified. The restitution properties of action potential duration at $90 \%$ repolarisation $\left(\mathrm{APD}_{90}\right)$ were also computed.

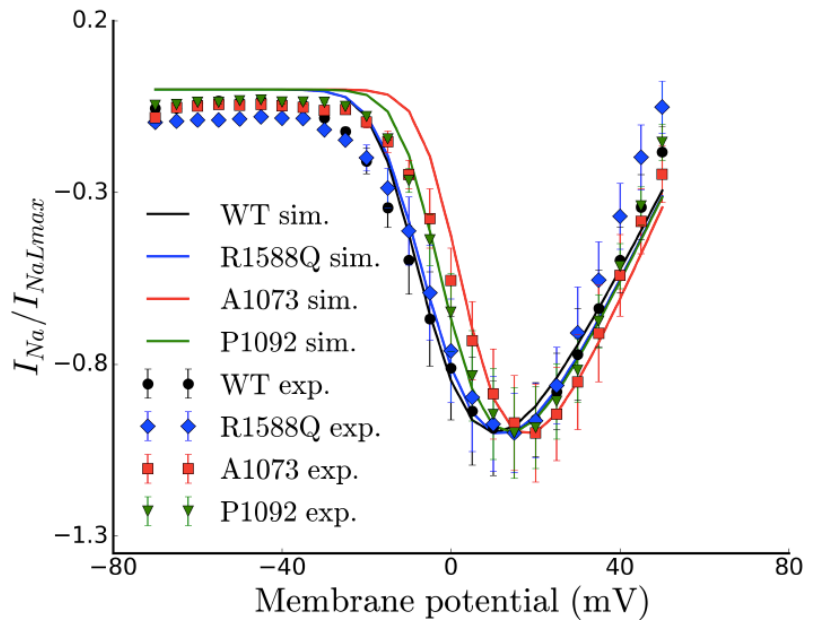

Figure 1. Simulated and experimental I-V curves of $I_{N a L}$ normalised to the maximal peak currents. Symbol with error bars represent the experimental data from [10] whilst lines show the simulation results.

\section{Results}

\subsection{Effects of SCN10A mutations on action potential}

The effects of the mutations on AP and corresponding time course of other ion channels during AP are shown in
Figure 2. The action potential duration (APD) was prolonged by $\mathrm{P} 1092$ and A1073 mutations more than R1588Q mutation at a BCL of $1000 \mathrm{~ms}$ (Figure 2). The SCN10A mutations slowed down the AP repolarisation in phase 1 and phase 2, resulting in an elevated plateau potential to more positive value (from $3 \mathrm{mV}$ for WT to 8 and $9 \mathrm{mV}$ for A1073 and P1092 respectively). The computed $\mathrm{APD}_{90}$ and plateau potential values of the simulated AP for the WT and SCN10A mutations are summurised in Figure 3.

Also, P1092 and A1073 mutations increased the intracellular calcium concentrations $\left[\mathrm{Ca}^{2+}\right]_{i}$ compared with WT. This indirect effect is due to the reverse mode of $\mathrm{Na}^{+}-\mathrm{Ca}^{2+}$ exchanger with $\mathrm{Ca}^{2+}$ actually entering the cell to recover more $\mathrm{Na}^{+}$entered through $\mathrm{Na}^{+}$channels. The main function of $\mathrm{Na}^{+}-\mathrm{Ca}^{2+}$ exchanger is to remove $\mathrm{Ca}^{2+}$ from cardiac myocytes by using the electrochemical gradient of $\mathrm{Na}^{+}$(forward mode).

Increased intracellular $\mathrm{Ca}^{2+}$ can increase the probability of early after-depolarizations (EADs), therefore increase the risk of arrhythmia and could have effects on reentry mechanisms.

In addition, A0173/P1092 mutations exhibited an increase in the peak of sodium current $I_{N a P}$. However, no noticeable change was marked in R1588Q condition (Figure 2).
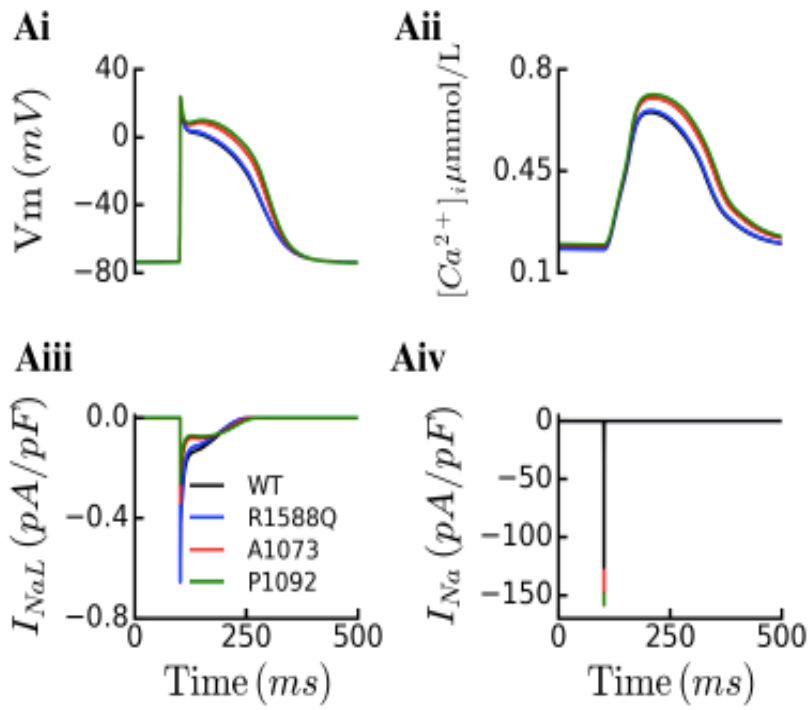

Figure 2. Effects of the SCN10A mutations on the AP of human atrial myocytes paced at $1 \mathrm{~Hz}$ (Ai), with the time course of intracellular $\left[\mathrm{Ca}^{2+}\right]_{\mathrm{i}}$ (Aii), and time course of current traces for $\mathrm{I}_{\mathrm{NaL}}$ (Aiii) and $\mathrm{I}_{\mathrm{Na}}$ (Aiv). 

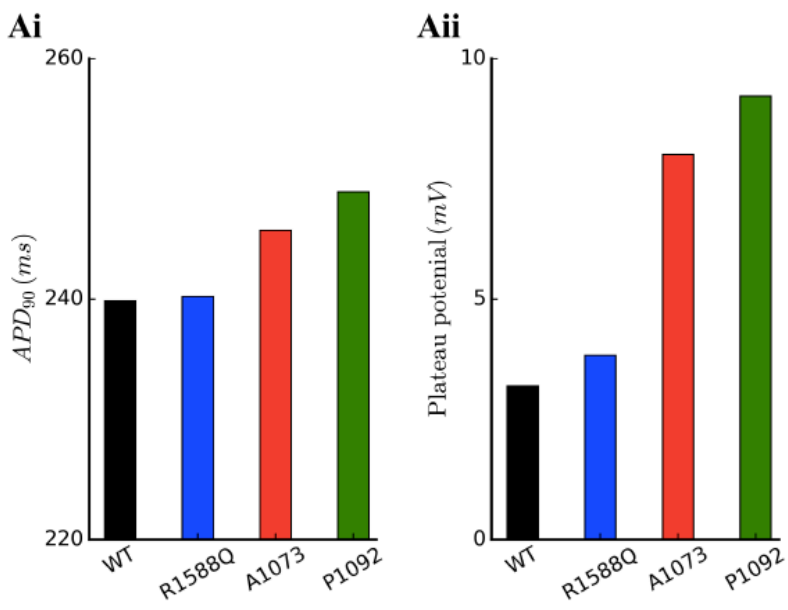

Figure 3. Summary of effect of the SCN10A mutations on the $\mathrm{APD}_{90}(\mathrm{~A})$ and plateau potential. (B). Plateau potential, quantified from the membrane potential within $10 \mathrm{~ms}$ to 50 ms after applying the external stimulus current.

\subsection{Effect of SCN10A mutations on APD restitution properties}

S1-S2 protocol was used in order to determine the effect of SCN10A mutations on the APD restitution properties of human cardiac myocytes. The model was paced at $1 \mathrm{~Hz}$ by a train of S1 stimuli. Then S2 stimulus was applied with a range of time interval delay.

The results illustrated that the gain-of-function mutations altered the rate dependent of APD and steepened the slop of APD restitution curves in the model compared with the WT condition (Figure 5). The maximum APD restitution slope was nearly unchanged for R1588Q variant but steepened for A1073 and P1092 conditions.
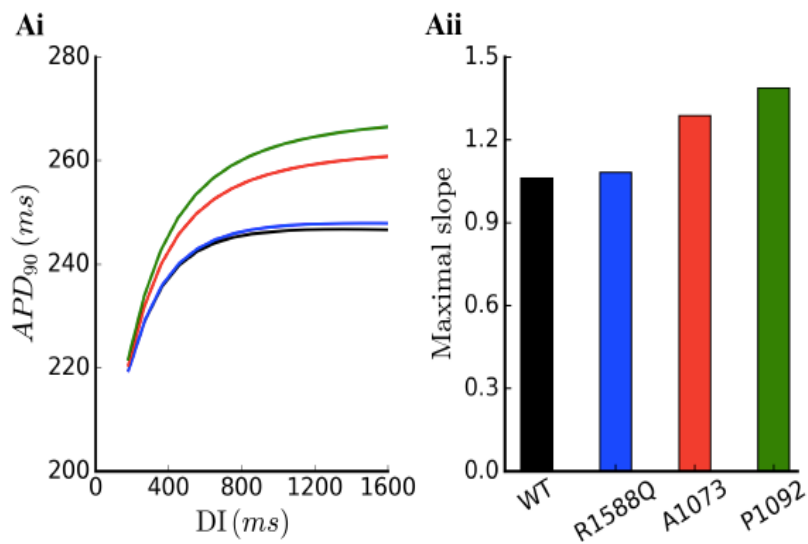

Figure 4: Simulated effects of SCN10A mutations on the APD restitution curves of human atrial myocytes. (Ai)
$\mathrm{APD}_{90}$ Restitution curves of WT and mutations. APD values plotted against the diastolic interval (DI). (Aii) Maximum slope of APD restitution curves.

\section{Discussion}

Colman et al. model of human atrial cells has been updated to incorporate the late sodium current $I_{N a L}$. The new model has been used to investigate the effect of SCN10A gene mutations on electrical function of human atrial cells.

Our results showed that $\mathrm{P} 1092$ and A1073 variants prolonged the $\mathrm{APD}_{90}$ which resulting in steepened the APD restitution curves with increased the maximal slope, whereas R1588Q restitution curve remined unchanged from the WT. The longer AP and increase intracellular sodium concentration $\mathrm{Na}^{+}$that cause $\mathrm{Ca}^{2+}$ overload in cardiomyocytes may increase the risk of AF [16,17].

In conclusion, the impact of SCN10A mutations on $\mathrm{APD}_{90}$ and the intracellular $\left[\mathrm{Ca}^{2+}\right]_{\mathrm{i}}$ could have important implications to understand the mechanisms behind which mutations enhance atrial function and influence susceptibility to AF.

\section{Acknowledgements}

This project was funded by Jazan University, Jazan, Kingdom in Saudi Arabia.

\section{References}

[1] Poulet C, Wettwer E, Grunnet M, Jespersen T, Fabritz L, Matschke $\mathrm{K}$, et al. Late sodium current in human atrial cardiomyocytes from patients in sinus rhythm and atrial fibrillation. PLoS One 2015;10:e0131432.

[2] Makielski JC. Late sodium current: A mechanism for angina, heart failure, and arrhythmia. Trends Cardiovasc. Med.2016;26:115-22.

[3] Belardinelli L, Liu G, Smith-Maxwell C, Wang W-Q, ElBizri N, Hirakawa R, et al. A novel, potent, and selective inhibitor of cardiac late sodium current suppresses experimental arrhythmias. J. Pharmacol. Exp. Ther. 2013;344:23-32.

[4] Trenor B, Cardona K, Gomez JF, Rajamani S, Ferrero JM, Belardinelli L, et al. Simulation and mechanistic investigation of the arrhythmogenic role of the late sodium current in human heart failure. PLoS One 2012;7:11-3.

[5] Karagueuzian HS, Pezhouman A, Angelini M, Olcese R. Enhanced late $\mathrm{Na}$ and $\mathrm{Ca}$ currents as effective antiarrhythmic drug targets. Front. Pharmacol. 2017;8:36.

[6] Ulbricht W. Sodium channel inactivation: molecular determinants and modulation. Physiol. Rev. 2005;85:1271301.

[7] Hong K, Xiong Q. Genetic basis of atrial fibrillation. Curr. Opin. Cardiol. 2014;29:220-6.

[8] Olesen MS, Nielsen MW, Haunsø S, Svendsen JH. Atrial fibrillation: the role of common and rare genetic variants. Eur. J. Hum. Genet. [Internet] 2014;22:297-306. 
[9] Lubitz SA, Yi BA, Ellinor PT. Genetics of atrial fibrillation. Heart Fail. Clin.2010;6:239-47.

[10]Ritchie MD, Denny JC, Zuvich RL, Crawford DC, Schildcrout JS, Bastarache L, et al. Genome- and phenomewide analyses of cardiac conduction identifies markers of arrhythmia risk. Circulation 2013;127:1377-85.

[11] Jabbari J, Olesen MS, Yuan L, Nielsen JB, Liang B, Macri $\mathrm{V}$, et al. Common and rare variants in SCN10A modulate the risk of atrial fibrillation. Circ. Cardiovasc. Genet. 2015;8:64-73.

[12] Grandi E, Pandit S V., Voigt N, Workman AJ, Dobrev D, Jalife J, et al. Human atrial action potential and $\mathrm{Ca} 2+$ model sinus rhythm and chronic atrial fibrillation. Circ. Res. 2011;109:1055-66.

[13] Ni H, Adeniran I, Zhang H. In-silico investigations of the functional impact of KCNA5 mutations on atrial mechanical dynamics. J. Mol. Cell. Cardiol. 2017.

[14] Horvath B, Bers DM. The late sodium current in heart failure: pathophysiology and clinical relevance. ESC Hear. Fail. 2014;1:26-40. sodium-calcium overload. Heart 2006;92 Suppl 4:iv1-iv5.

[16] i Q, Huang H, Liu G, Lam K, Rutberg J, Green MS, et al. Gain-of-function mutation of Nav1.5 in atrial fibrillation enhances cellular excitability and lowers the threshold for action potential firing. Biochem. Biophys. Res. Commun. 2009.

[17] Makiyama T, Akao M, Shizuta S, Doi T, Nishiyama K, Oka $Y$, et al. A Novel SCN5A gain-of-function mutation M1875T associated with familial atrial fibrillation. J. Am. Coll. Cardiol. 2008.

Address for correspondence.

Inas Ali Al Nemi.

Room 3.17, Schuster Building, University of Manchester, Manchester, UK,

M13 9PL.

inas.alnemi@postgrad.manchester.ac.uk

[15] Noble D, Noble PJ. Late sodium current in the pathophysiology of cardiovascular disease: Consequences of 